PROCEEDINGS OF THE

AMERICAN MATHEMATICAL SOCIETY

Volume 136, Number 10, October 2008, Pages 3383-3390

S 0002-9939(08)08959-4

Article electronically published on May 15, 2008

\title{
FLATNESS OF THE LINKED GRASSMANNIAN
}

\author{
DAVID HELM AND BRIAN OSSERMAN \\ (Communicated by Ted Chinburg)
}

\begin{abstract}
We show that the linked Grassmannian scheme, which arises in a functorial compactification of spaces of limit linear series, and in local models of certain Shimura varieties, is Cohen-Macaulay, reduced, and flat. We give an application to spaces of limit linear series.
\end{abstract}

\section{INTRODUCTION}

The linked Grassmannian was introduced in 9 as a tool for the construction of a moduli scheme of limit linear series, providing a functorial description which also compactified the spaces introduced by Eisenbud and Harris in [3. It also arises in other contexts: for instance, the length 2 case over $\mathbb{Z}_{p}$ gives a local model for certain unitary Shimura varieties 4 .

A few basic properties of the linked Grassmannian were proved in [9], but only as much as was necessary for the application to limit linear series. In particular, although the dimensions were computed, a substantial question that remained open (Question A.18 of loc. cit.) was the flatness of linked Grassmannian schemes over their base. In this paper, we address this question. We perform a detailed analysis in the case of linked Grassmannians of length 2, giving a local description at each point (Theorem 3.2 below). This description allows us to invoke the work of Zhang 11 to conclude that the linked Grassmannians are Cohen-Macaulay, from which we conclude flatness, as well as reducedness. Finally, we use an inductive argument to conclude the same statements for linked Grassmannians of arbitrary length (Theorem 4.2).

As consequences, we are able to conclude (Theorem 4.4) that when a limit linear series space has the expected dimension, it is flat over its base and Cohen-Macaulay. We also see that the linked Grassmannian itself provides a new class of flat degenerations of the Grassmannian; such degenerations have frequently been of interest in the combinatorial study of the Grassmannian, and the linked Grassmannian thus provides a potentially useful new tool for such analysis.

There is some substantial overlap of our results, in the special case of length 2 over $\mathbb{Z}_{p}$, with the reducedness and flatness results of Goertz [4, in particular section 4.4.5 and Theorem 4.12. However, because we are working over a more general base,

Received by the editors May 16, 2006, and, in revised form, August 16, 2006.

2000 Mathematics Subject Classification. Primary 14M15; Secondary 14H51, 14 G35.

The first author was supported by a fellowship from the NSF.

The second author was supported by fellowships from the Clay Mathematics Institute and the NSF during the preparation of this paper.

(C)2008 American Mathematical Society Reverts to public domain 28 years from publication 
and our local calculation is rather concise and gives a stronger Cohen-Macaulayness result, we have chosen to keep our presentation self-contained.

\section{REVIEW OF THE Linked GRASSMANNiAN}

We briefly review the basic definitions and results of the linked Grassmannian.

Definition 2.1. Let $S$ be an integral, locally Cohen-Macaulay scheme, and let $\mathscr{E}_{1}, \ldots, \mathscr{E}_{n}$ be vector bundles on $S$, each of rank $d$. Given maps $f_{i}: \mathscr{E}_{i} \rightarrow \mathscr{E}_{i+1}$ and $g_{i}: \mathscr{E}_{i+1} \rightarrow \mathscr{E}_{i}$, for $1 \leq i \leq n-1$, and a positive integer $r<d$, we denote by $\mathcal{L G}:=\mathcal{L G}\left(r,\left\{\mathscr{E}_{i}\right\}_{i},\left\{f_{i}, g_{i}\right\}_{i}\right)$ the functor associating to each $S$-scheme $T$ the set of sub-bundles $V_{1}, \ldots, V_{n}$ of $\mathscr{E}_{1, T}, \ldots, \mathscr{E}_{n, T}$ having rank $r$ and satisfying $f_{i, T}\left(V_{i}\right) \subset$ $V_{i+1}, g_{i, T}\left(V_{i+1}\right) \subset V_{i}$ for all $i$ between 1 and $n-1$.

We say that $\mathcal{L G}$ is a linked Grassmannian functor if the following further conditions on the $f_{i}$ and $g_{i}$ are satisfied:

(I) There exists some $s \in \mathscr{O}_{S}$ such that $f_{i} g_{i}=g_{i} f_{i}$ is scalar multiplication by $s$ for all $i$.

(II) Wherever $s$ vanishes, the kernel of $f_{i}$ is precisely equal to the image of $g_{i}$, and vice versa. More precisely, for any $i$ and given any two integers $r_{1}$ and $r_{2}$ such that $r_{1}+r_{2}<d$, then the closed subscheme of $S$ obtained as the locus where $f_{i}$ has rank less than or equal to $r_{1}$ and $g_{i}$ has rank less than or equal to $r_{2}$ is empty.

(III) At any point of $S, \operatorname{im} f_{i} \cap \operatorname{ker} f_{i+1}=0$, and $\operatorname{im} g_{i+1} \cap \operatorname{ker} g_{i}=0$. More precisely, for any integer $r_{1}$, and any $i$, we have locally closed subschemes of $S$ corresponding to the locus where $f_{i}$ has rank exactly $r_{1}$, and $f_{i+1} f_{i}$ has rank less than or equal to $r_{1}-1$, and similarly for the $g_{i}$. Then we require simply that all of these subschemes be empty.

The main theorem of [9] on the linked Grassmannian is the following:

Theorem 2.2 ([9, Lem. A.3, Lem. A.12, Thm. A.15]). $\mathcal{L G}$ is representable by a scheme $L G$; this scheme is naturally a closed subscheme of the obvious product $G_{1} \times$ $\cdots \times G_{n}$ of Grassmannian schemes over $S$, which is smooth of relative dimension $n r(d-r)$. Each component of $L G$ has codimension $(n-1) r(d-r)$ in the product and maps surjectively to $S$; in fact, the smooth points of $L G$ are dense in every component of every fiber. If $s$ is non-zero, then $L G$ is also irreducible.

\section{The MAin CALCULATiON}

We now give an explicit local computation of the structure of a linked Grassmannian of length 2 .

Notation 3.1. Let $M_{d, S}(2, s)$ be the affine scheme over $S$ parametrizing pairs of $d \times d$ matrices $M, N$ with coefficients in $\mathscr{O}_{S}$ and with $M N=N M=s$.

Theorem 3.2. Fix vector bundles $\mathscr{E}_{1}, \mathscr{E}_{2}$ of rank $d$ on $S$, maps $f: \mathscr{E}_{1} \rightarrow \mathscr{E}_{2}$ and $g: \mathscr{E}_{2} \rightarrow \mathscr{E}_{1}, r \in \mathbb{N}$ and $s \in \mathscr{O}_{S}$ satisfying the conditions for a linked Grassmannian of length 2. Then given any field $k$ and a $k$-valued point $x=\left(V_{1}, V_{2}\right) \in L G:=$ $L G\left(r,\left\{\mathscr{E}_{1}, \mathscr{E}_{2}\right\},\{f, g\}\right)$, there exists a Zariski neighborhood $U$ of $x$ in $L G$ and an isomorphism

$$
U \stackrel{\sim}{\rightarrow} \mathbb{A}_{S^{\prime}}^{m} \times M_{\ell, S^{\prime}}(2, s)
$$


sending $x$ to the origin, where $S^{\prime} \subseteq S$ is the image of $U$,

$$
\ell= \begin{cases}r-\operatorname{dim}\left(g\left(V_{2}\right)\right)-\operatorname{dim}\left(f\left(V_{1}\right)\right): & s=0 \text { at } x, \\ 0: & s \neq 0 \text { at } x,\end{cases}
$$

and $m=r(d-r)-\ell^{2}$.

Proof. The question is local on the base, so we may assume that $S=\operatorname{Spec} R$ and that $\mathscr{E}_{1}$ and $\mathscr{E}_{2}$ are trivial bundles. Furthermore, if $s \neq 0$ at $x$, then $L G$ is locally isomorphic simply to the Grassmannian $G(r, d)$, so in a neighborhood of $x$ we find that $L G$ is isomorphic to $\mathbb{A}_{S}^{r(d-r)}$, as asserted. So we may suppose that $s=0$ at $x$.

Let $\bar{x}$ be the image of $x$ under the structure morphism from $L G$ to $S$. Then the spaces $\left(V_{1}, V_{2}\right)$ determining $x$ are naturally subspaces of $\bar{x}^{*} \mathscr{E} 1$ and $\bar{x}^{*} \mathscr{E} 2$, and must satisfy $f\left(V_{1}\right) \subseteq V_{2}$ and $g\left(V_{2}\right) \subseteq V_{1}$. Let $c$ be the dimension of the kernel of $f$ on $\bar{x}^{*} \mathscr{E}_{1}$, and let $d_{1}$ and $d_{2}$ be the dimensions of $f\left(V_{1}\right)$ and $g\left(V_{2}\right)$, respectively.

We make the following choices:

- $\left\{\alpha_{1}^{1}, \ldots, \alpha_{d_{1}}^{1}\right\}$ in $V_{1}$ so that $\left\{f\left(\alpha_{1}^{1}\right), \ldots, f\left(\alpha_{d_{1}}^{1}\right)\right\}$ is a basis for $f\left(V_{1}\right)$;

- $\left\{\beta_{1}^{1}, \ldots, \beta_{d_{2}}^{1}\right\}$ in $V_{2}$ so that $\left\{g\left(\beta_{1}^{1}\right), \ldots, g\left(\beta_{d_{2}}^{1}\right)\right\}$ is a basis for $g\left(V_{2}\right)$;

- $\left\{\alpha_{1}^{2}, \ldots, \alpha_{r-d_{1}-d_{2}}^{2}\right\}$ in $\bar{x}^{*} \mathscr{E}_{1}$ so that $\left\{f\left(\alpha_{1}^{2}\right), \ldots, f\left(\alpha_{r-d_{1}-d_{2}}^{2}\right)\right\}$ extends the set $\left\{f\left(\alpha_{1}^{1}\right), \ldots, f\left(\alpha_{d_{1}}^{1}\right)\right\}$ to a basis for $\operatorname{ker} g \cap V_{2}$;

- $\left\{\beta_{1}^{2}, \ldots, \beta_{r-d_{1}-d_{2}}^{2}\right\}$ in $\bar{x}^{*} \mathscr{E}_{2}$ so that $\left\{g\left(\beta_{1}^{2}\right), \ldots, g\left(\beta_{r-d_{1}-d_{2}}^{2}\right)\right\}$ extends the set $\left\{g\left(\beta_{1}^{1}\right), \ldots, g\left(\beta_{d_{2}}^{1}\right)\right\}$ to a basis for $\operatorname{ker} f \cap V_{1}$,

- $\left\{\alpha_{1}^{3}, \ldots, \alpha_{d-r-c+d_{2}}^{3}\right\}$ in $\bar{x}^{*} \mathscr{E}_{1}$ so that $\left\{f\left(\alpha_{1}^{3}\right), \ldots, f\left(\alpha_{d-r-c-d_{2}}^{3}\right)\right\}$ extends the set $\left\{f\left(\alpha_{1}^{1}\right), \ldots, f\left(\alpha_{d_{1}}^{1}\right), f\left(\alpha_{1}^{2}\right), \ldots, f\left(\alpha_{r-d_{1}-d_{2}}^{2}\right)\right\}$ to a basis for ker $g$, and

- $\left\{\beta_{1}^{3}, \ldots, \beta_{d_{1}+c-r}^{3}\right\}$ in $\bar{x}^{*} \mathscr{E}_{2}$ so that $\left\{g\left(\beta_{1}^{3}\right), \ldots, g\left(\beta_{d_{1}+c-r}^{3}\right)\right\}$ extends the set $\left\{g\left(\beta_{1}^{1}\right), \ldots, g\left(\beta_{d_{2}}^{1}\right), g\left(\beta_{1}^{2}\right), \ldots, g\left(\beta_{r-d_{1}-d_{2}}^{2}\right)\right\}$ to a basis for $\operatorname{ker} f$.

We lift each $\alpha_{j}^{i}$ defined above to a section $\tilde{\alpha}_{j}^{i}$ of $\mathscr{E}_{1}$, and each $\beta_{j}^{i}$ to a section $\tilde{\beta}_{j}^{i}$ of $\mathscr{E}_{2}$. These lifts allow us to define nice bases for these bundles in a neighborhood of $\bar{x}$. Specifically, for $i$ between 1 and $d$, we define $e_{i}^{1}$ and $e_{i}^{2}$ as follows:

- $e_{1}^{1}, \ldots, e_{d_{1}}^{1}:=\tilde{\alpha}_{1}^{1}, \ldots, \tilde{\alpha}_{d_{1}}^{1}$

- $e_{d_{1}+1}^{1}, \ldots, e_{r-d_{2}}^{1}:=g\left(\tilde{\beta}_{1}^{2}\right), \ldots, g\left(\tilde{\beta}_{r-d_{1}-d_{2}}^{2}\right)$;

- $e_{r-d_{2}+1}^{1}, \ldots, e_{r}^{1}:=g\left(\tilde{\beta}_{1}^{1}\right), \ldots, g\left(\tilde{\beta}_{d_{2}}^{1}\right)$;

- $e_{r+1}^{1}, \ldots, e_{2 r-d_{1}-d_{2}}^{1}:=\tilde{\alpha}_{1}^{2}, \ldots, \tilde{\alpha}_{r-d_{1}-d_{2}}^{2}$;

- $e_{2 r-d_{1}-d_{2}+1}^{1}, \ldots, e_{d+r-d_{1}-c}^{1}:=\tilde{\alpha}_{1}^{3}, \ldots, \tilde{\alpha}_{d-r-c+d_{2}}^{3}$;

- $e_{d+r-d_{1}-c+1}^{1}, \ldots, e_{d}^{1}:=g\left(\tilde{\beta}_{1}^{3}\right), \ldots, g\left(\tilde{\beta}_{d_{1}+c-r}^{3}\right)$;

- $e_{1}^{2}, \ldots, e_{d_{1}}^{2}:=f\left(\tilde{\alpha}_{1}^{1}\right), \ldots, f\left(\tilde{\alpha}_{d_{1}}^{1}\right)$;

- $e_{d_{1}+1}^{2}, \ldots, e_{r-d_{2}}^{2}:=f\left(\tilde{\alpha}_{1}^{2}\right), \ldots, f\left(\tilde{\alpha}_{r-d_{1}-d_{2}}^{2}\right)$;

- $e_{r-d_{2}+1}^{2}, \ldots, e_{r}^{2}:=\tilde{\beta}_{1}^{1}, \ldots, \tilde{\beta}_{d_{2}}^{1}$;

- $e_{r+1}^{2}, \ldots, e_{2 r-d_{1}-d_{2}}^{2}:=\tilde{\beta}_{1}^{2}, \ldots, \tilde{\beta}_{r-d_{1}-d_{2}}^{2}$;

- $e_{2 r-d_{1}-d_{2}+1}^{2}, \ldots, e_{d+r-d_{1}-c}^{2}:=f\left(\tilde{\alpha}_{1}^{3}\right), \ldots, f\left(\tilde{\alpha}_{d-r-c+d_{2}}^{3}\right)$;

- $e_{d+r-d_{1}-c}^{2}, \ldots, e_{d}^{2}:=\tilde{\beta}_{1}^{3}, \ldots, \tilde{\beta}_{d_{1}+c-r}^{3}$.

Then $e_{1}^{1}, \ldots, e_{d}^{1}$ and $e_{1}^{2}, \ldots, e_{d}^{2}$ restrict to bases for $\bar{x}^{*} \mathscr{E}_{1}$ and $\bar{x}^{*} \mathscr{E}_{2}$, respectively. Shrinking $S$ to a smaller neighborhood of $\bar{x}$, we can thus assume that $e_{1}^{1}, \ldots, e_{d}^{1}$ is a basis for $\mathscr{E}_{1}$ and $e_{1}^{2}, \ldots, e_{d}^{2}$ is a basis for $\mathscr{E}_{2}$. 
With respect to these bases, the maps $f$ and $g$ have block forms

$$
\begin{aligned}
f & =\left(\begin{array}{cccccc}
\operatorname{Id}_{d_{1}} & 0 & 0 & 0 & 0 & 0 \\
0 & 0 & 0 & \operatorname{Id}_{r-d_{1}-d_{2}} & 0 & 0 \\
0 & 0 & s \operatorname{Id}_{d_{2}} & 0 & 0 & 0 \\
0 & s \mathrm{Id}_{r-d_{1}-d_{2}} & 0 & 0 & 0 & 0 \\
0 & 0 & 0 & 0 & \operatorname{Id}_{d-r-c+d_{2}} & 0 \\
0 & 0 & 0 & 0 & 0 & s \mathrm{Id}_{d_{1}+c-r}
\end{array}\right), \\
g & =\left(\begin{array}{cccccc}
s \mathrm{Id}_{d_{1}} & 0 & 0 & 0 & 0 & 0 \\
0 & 0 & 0 & \mathrm{Id}_{r-d_{1}-d_{2}} & 0 & 0 \\
0 & 0 & \operatorname{Id}_{d_{2}} & 0 & 0 & 0 \\
0 & s \mathrm{Id}_{r-d_{1}-d_{2}} & 0 & 0 & 0 & 0 \\
0 & 0 & 0 & 0 & s \operatorname{Id}_{d-r-c+d_{2}} & 0 \\
0 & 0 & 0 & 0 & 0 & \operatorname{Id}_{d_{1}+c-r}
\end{array}\right) .
\end{aligned}
$$

If we consider $L G$ as a subscheme of $G(r, d)_{R} \times{ }_{R} G(r, d)_{R}$ in the obvious way, $x$ has an affine neighborhood in this product given by a pair of $d-r$ by $r$ matrices $A^{1}, A^{2}$, where this pair corresponds to the pair of subspaces $\left(V_{1}^{\prime}, V_{2}^{\prime}\right)$ satisfying

$$
\begin{aligned}
& V_{1}^{\prime}=\operatorname{colspan}\left(\begin{array}{c}
\mathrm{Id}_{r} \\
A^{1}
\end{array}\right), \\
& V_{2}^{\prime}=\operatorname{colspan}\left(\begin{array}{c}
\mathrm{Id}_{r} \\
A^{2}
\end{array}\right) .
\end{aligned}
$$

We wish to find equations for the intersection of this neighborhood with $L G$. A pair $\left(A^{1}, A^{2}\right)$ will lie in $L G$ if and only if $f\left(V_{1}^{\prime}\right)$ is contained in $V_{2}^{\prime}$ and $g\left(V_{2}^{\prime}\right)$ is contained in $V_{1}^{\prime}$. To write these conditions explicitly, we break the matrices defining $V_{1}^{\prime}$ and $V_{2}^{\prime}$ into block form as follows:

$$
V_{1}^{\prime}=\operatorname{colspan}\left(\begin{array}{ccc}
\operatorname{Id}_{d_{1}} & 0 & 0 \\
0 & \operatorname{Id}_{r-d_{1}-d_{2}} & 0 \\
0 & 0 & \operatorname{Id}_{d_{2}} \\
A_{11}^{1} & A_{12}^{1} & A_{13}^{1} \\
A_{21}^{1} & A_{22}^{1} & A_{23}^{1} \\
A_{31}^{1} & A_{32}^{1} & A_{33}^{1}
\end{array}\right)
$$

and similarly for $A^{2}$. Here the horizontal divisions of $A$ are chosen so that the block division above corresponds to the block division for the matrices for $f$ and $g$.

Now we have:

$$
f\left(V_{1}^{\prime}\right)=\operatorname{colspan}\left(\begin{array}{ccc}
\operatorname{Id}_{d_{1}} & 0 & 0 \\
A_{11}^{1} & A_{12}^{1} & A_{13}^{1} \\
0 & 0 & s \operatorname{Id}_{d_{2}} \\
0 & s \operatorname{Id}_{r-d_{1}-d_{2}} & 0 \\
A_{21}^{1} & A_{22}^{1} & A_{23}^{1} \\
s A_{31}^{1} & s A_{32}^{1} & s A_{33}^{1}
\end{array}\right),
$$

and it is easy to see that this is contained in $V_{2}^{\prime}$ if and only if the matrix identity

$$
\left(\begin{array}{ccc}
A_{11}^{2} & A_{12}^{2} & A_{13}^{2} \\
A_{21}^{2} & A_{22}^{2} & A_{23}^{2} \\
A_{31}^{2} & A_{32}^{2} & A_{33}^{2}
\end{array}\right)\left(\begin{array}{ccc}
\operatorname{Id}_{d_{1}} & 0 & 0 \\
A_{11}^{1} & A_{12}^{1} & A_{13}^{1} \\
0 & 0 & s \operatorname{Id}_{d_{2}}
\end{array}\right)=\left(\begin{array}{ccc}
0 & s \operatorname{Id}_{r-d_{1}-d_{2}} & 0 \\
A_{21}^{1} & A_{22}^{1} & A_{23}^{1} \\
s A_{31}^{1} & s A_{32}^{1} & s A_{33}^{1}
\end{array}\right)
$$


is satisfied. Similarly, $g\left(V_{2}^{\prime}\right)$ is contained in $V_{1}^{\prime}$ if and only if we have

$$
\left(\begin{array}{ccc}
A_{11}^{1} & A_{12}^{1} & A_{13}^{1} \\
A_{21}^{1} & A_{22}^{1} & A_{23}^{1} \\
A_{31}^{1} & A_{32}^{1} & A_{33}^{1}
\end{array}\right)\left(\begin{array}{ccc}
s \operatorname{Id}_{d_{1}} & 0 & 0 \\
A_{11}^{2} & A_{12}^{2} & A_{13}^{2} \\
0 & 0 & \operatorname{Id}_{d_{2}}
\end{array}\right)=\left(\begin{array}{ccc}
0 & s \operatorname{Id}_{r-d_{1}-d_{2}} & 0 \\
s A_{21}^{2} & s A_{22}^{2} & s A_{23}^{2} \\
A_{31}^{2} & A_{32}^{2} & A_{33}^{2}
\end{array}\right) .
$$

If we solve for the matrix variables $A_{13}^{1}, A_{23}^{1}, A_{31}^{2}, A_{32}^{2}, A_{33}^{2}, A_{11}^{2}, A_{21}^{2}$, and $A_{22}^{1}$ in terms of the remaining variables, all of the above relations disappear except for the relations

and the result follows.

$$
A_{12}^{1} A_{12}^{2}=A_{12}^{2} A_{12}^{1}=s \operatorname{Id}_{r-d_{1}-d_{2}},
$$

\section{Conclusion of the MAin theOrems}

Zhang 11] has shown, using the theory of Hodge algebras of de Concini, Eisenbud and Procesi 1, that spaces such as those arising in Theorem 3.2 are CohenMacaulay. In particular, we have:

Theorem 4.1 (Zhang). Let $k$ be a field. Then $M_{\ell, k}(2,0)$ is Cohen-Macaulay.

Proof. This is [11, Thm. 22]. Since this reference is difficult to obtain, we are grateful to the referee for explaining the following alternative argument:

Let $W\left(k_{1}, k_{2}\right)$ denote the closed subscheme of $M_{\ell, k}(2,0)$ consisting of those pairs of $\ell \times \ell$ matrices $M, N$ such that $M N=N M=0, M$ has rank at most $k_{1}$, and $N$ has rank at most $k_{2}$. Strickland [10, p. 524, §2] has shown that the $W\left(k_{1}, k_{2}\right)$ are reduced, and when $k_{1}+k_{2} \leq \ell$, that they are also Cohen-Macaulay. Setting $k_{1}=k_{2}=\ell$ in the first result, we see in particular that $M_{\ell, k}(2,0)$ is reduced, hence scheme-theoretically the union of $W(j, \ell-j)$ for $j=0, \ldots, \ell$.

Define

$$
W^{i}:=\bigcup_{j=0}^{i} W(j, \ell-j)
$$

and observe that

$$
W^{i-1} \cap W(i, \ell-i)=W(i-1, \ell-i)
$$

(scheme-theoretically). Moreover, $W(i-1, \ell-i)$ has codimension one in both $W^{i-1}$ and $W(i, \ell-i)$. Thus by Goertz [4, Lemma 4.22], if $W^{i-1}$ is Cohen-Macaulay, then so is $W^{i}$. By induction, we find that $W^{\ell}$ is Cohen-Macaulay. But $W^{\ell}$ is equal to $M_{\ell, k}(2,0)$.

We are thus able to show the following.

Theorem 4.2. Suppose that $S$ is integral and Cohen-Macaulay, and let $L G:=$ $L G\left(r,\left\{\mathscr{E}_{i}\right\}_{i},\left\{f_{i}, g_{i}\right\}_{i}\right)$ be any linked Grassmannian scheme over $S$. Then $L G$ is flat over $S$, and reduced and Cohen-Macaulay, with reduced fibers.

Proof. We first show that $L G$ is Cohen-Macaulay whenever $S=\operatorname{Spec} k$. We prove this by induction on $n$, starting with the case $n=2$. In this case, by Theorem 3.2 we can conclude that $L G$ is Cohen-Macaulay if we know that $M_{\ell, S}(2, s)$ is CohenMacaulay. If $s \neq 0$, we have $\ell=0$, and $M_{\ell, S}(2, s)$ is just a point. If $s=0$, we have the space of pairs of $\ell \times \ell$ matrices $M, N$ with coefficients in $k$ and with $M N=N M=0$. By Theorem 4.1, this space is Cohen-Macaulay.

We now induct on $n$. We let $L G$ be a linked Grassmannian of length $n$, and denote by $L G_{n-1}$ (respectively, $L G^{\prime}$ ) the truncated linked Grassmannians of length 
$n-1$ (respectively, 2) obtained from $L G$ by forgetting $\mathscr{E}_{n}, f_{n}, g_{n}$ (respectively, $\mathscr{E}_{i}, f_{i}, g_{i}$ for all $\left.i<n-1\right)$. Then $L G_{n-1}$ naturally lies in the product of Grassmannians $G_{n-1}:=G\left(r, \mathscr{E}_{1}\right) \times \cdots \times G\left(r, \mathscr{E}_{n-1}\right)$, and $L G^{\prime}$ in $G^{\prime}:=G\left(r, \mathscr{E}_{n-1}\right) \times G\left(r, \mathscr{E}_{n}\right)$. Let

$$
\Delta: G:=G\left(r, \mathscr{E}_{1}\right) \times \cdots \times G\left(r, \mathscr{E}_{n}\right) \rightarrow G_{n-1} \times G^{\prime}
$$

be the map which doubles the $(n-1)$ st coordinate; it is then clear by considering the functors of points that we have that $L G \subseteq G$ is the fiber product of $\Delta$ with the inclusion map $L G_{n-1} \times L G^{\prime} \rightarrow G_{n-1} \times G^{\prime}$.

Now, the image of $\Delta$ is a locally complete intersection of codimension $r(d-r)$ in $G_{n-1} \times G^{\prime}$, so we conclude that $L G$ is cut out by $r(d-r)$ equations inside of $L G_{n-1} \times L G^{\prime}$, and since this is also the codimension of $L G$, we find that the induction hypothesis that $L G_{n-1}$ and $L G^{\prime}$ are Cohen-Macaulay then implies that $L G$ is Cohen-Macaulay as well. This shows that $L G$ is always Cohen-Macaulay over Spec $k$.

Next, still assuming that $S=\operatorname{Spec} k$, by Theorem 2.2, we have that $L G$ is smooth and hence reduced on an open dense subset, and since $L G$ is Cohen-Macaulay, it cannot have imbedded components, and we conclude that $L G$ is also reduced as long as the base is a point.

Finally, suppose $S$ is an arbitrary integral, Cohen-Macaulay scheme. The base change of $L G$ to any integral, Cohen-Macaulay scheme is another linked Grassmannian, so since we have reducedness when $S=\operatorname{Spec} k$, we have that condition (i) of the following lemma is satisfied for any base. Similarly, by Theorem 2.2 every topological component of $L G$ surjects onto the base after base change to Spec $A$ with $A$ a DVR, and condition (ii) of the following lemma is satisfied. Thus, the lemma implies that any linked Grassmannian is reduced and flat. Finally, we can conclude Cohen-Macaulayness for arbitrary $S$ by [7, Cor., p. 181].

Lemma 4.3. Let $f: X \rightarrow S$ be a morphism locally of finite presentation with $S$ reduced and Noetherian, and suppose that:

(i) the fibers of $f$ are geometrically reduced;

(ii) after arbitrary base change to Spec $A$, with $A$ a DVR, no (topological) component of $X \times_{S} \operatorname{Spec} A$ is supported over the special fiber.

Then $X$ is reduced, and $f$ is flat.

Proof. By [6, Thm. 11.8.1], we can prove that $f$ is flat by checking that it is flat after arbitrary base change under morphisms Spec $A \rightarrow S$, where $A$ is a DVR. Since $f$ has geometrically reduced fibers, the fibers remain reduced under base change, and assumption (ii) allows us to conclude that $\left(X \times{ }_{S} \operatorname{Spec} A\right)_{\text {red }}$ is flat over $\operatorname{Spec} A$ by [6. Prop. 14.3.8].

It then follows by [9, Lem. 6.13] that $X \times_{S} \mathrm{Spec}_{A}$ is reduced, hence flat, giving us that $f$ is flat. The flatness of $f$ then also implies by [5, Cor. 3.3.5] that $X$ is reduced, as desired.

Finally, we use the previous theorem to conclude a similar flatness result for the limit linear series schemes introduced in 9 ; see that paper for the appropriate definitions.

Theorem 4.4. Fix integers $r, d, n$, and ramification sequences $\alpha^{1}, \ldots, \alpha^{n}$, and let $X_{0}$ be a curve of compact type over Spec $k$ having no more than two components, with marked points $P_{1}, \ldots, P_{n}$. Suppose that the space $G_{d}^{r}\left(X_{0}\right):=G_{d}^{r}\left(X_{0} / k\right.$; 
$\left.\left\{\left(P_{i}, \alpha^{i}\right)\right\}_{i}\right)$ of limit linear series on $X_{0}$ of degree $d$ and dimension $r$, with prescribed ramification at the $P_{i}$, has exactly the expected dimension $\rho:=(r+1)(d-r)-r g-$ $\sum_{i, j} \alpha_{j}^{i}$.

Then $G_{d}^{r}\left(X_{0}\right)$ is Cohen-Macaulay, and if we have a smoothing family of curves $X / B,\left\{\tilde{P}_{i}\right\}_{i}$ (see [9, Def. 3.1]) where $X_{0},\left\{P_{i}\right\}_{i}$ occurs as a fiber over $b_{0} \in B$, then $G_{d}^{r}(X):=G_{d}^{r}\left(X / B ;\left\{\left(\tilde{P}_{i}, \alpha^{i}\right)\right\}_{i}\right)$, the relative space of limit linear series on $X / B$, is Cohen-Macaulay and flat over $B$ at every point over $b_{0}$.

We remark that the expected dimension hypothesis is satisfied for a general curve with marked points in characteristic 0; see [8].

Proof. Indeed, in the proof of [9. Thm. 5.3] the space $G_{d}^{r}(X)$ of limit linear series on a smoothing family $X / B$ (where we may allow $B=\operatorname{Spec} k, X=X_{0}$ ) is constructed inside a product of Grassmannians by intersecting a linked Grassmannian with a finite collection of Schubert cycles, and this has dimension $\rho$ in the fiber over $b_{0}$ if and only if this intersection has maximal codimension over $b_{0}$. Since we now know that linked Grassmannians are Cohen-Macaulay, at any point over $b_{0}$ we have a proper-dimensional intersection of Cohen-Macaulay schemes inside a regular one, and this must be Cohen-Macaulay by the lemma below. Since $B$ is regular as part of the hypothesis of a smoothing family, we also obtain that $G_{d}^{r}(X)$ is flat over $B$ (at least, at any point over $b_{0}$ ) by [7, Thm. 23.1].

We recall the following well-known lemma and include the proof for lack of a suitable reference:

Lemma 4.5. Let $X$ be regular, and $Y_{1}, \ldots, Y_{n}$ closed subschemes of $X$, each CohenMacaulay, of codimensions $c_{1}, \ldots, c_{n}$. Let $Z=Y_{1} \cap \cdots \cap Y_{n}$, and suppose that $Z$ has codimension $\sum_{i} c_{i}$ in $X$. Then $Z$ is also Cohen-Macaulay.

Proof. By induction, it is clearly enough to consider the case that $n=2$. Also, the question is purely local, so we are reduced to the following: let $R$ be a regular local ring, and $I, J$ ideals of codimension $d$, e such that $R / I$ and $R / J$ are Cohen-Macaulay, and $I+J$ has codimension $d+e$. Then we want to see that $R /(I+J)$ is Cohen-Macaulay. By [2, Cor. 19.15], the Cohen-Macaulayness of the rings $R / I, R / J, R /(I+J)$ is equivalent to having free resolutions over $R$ of lengths $d, e, d+e$ respectively. Thus, we may assume we have free resolutions $\mathscr{F} \bullet, \mathscr{G}^{\bullet}$ of $R / I$ and $R / J$ having lengths $d, e$ respectively. We claim that the tensor complex $\mathscr{F} \bullet \otimes \mathscr{G} \bullet$, which has length $d+e$, is still exact, thus giving a free resolution of $R /(I+J)$ of length $d+e$ and showing that $R /(I+J)$ is Cohen-Macaulay.

By [2, Thm. 20.9], it is enough to check that $\mathscr{F} \bullet \otimes \mathscr{G} \bullet$ is exact after localizing at an arbitrary prime $\mathfrak{p}$ of codimension strictly less than $d+e$ : indeed, condition (1) of loc. cit. is automatically satisfied for the tensor product of two free resolutions, and to verify (2), if we had (in the notation of loc. cit.) depth $I\left(\varphi_{k}\right)<k$ for some $k \leq d+e$, we could choose $\mathfrak{p}$ to be a minimal prime of $I\left(\varphi_{k}\right)$ having codimension strictly less than $d+e$, and (2) would still be violated after localizing at $\mathfrak{p}$.

Now, let $\mathfrak{p}$ be arbitrary of codimension less than $d+e$. It cannot contain $I+J$ and thus does not contain both $I$ and $J$; without loss of generality, say that $I$ is not contained in $\mathfrak{p}$. But if we localize $\mathscr{F}^{\bullet}$ at $\mathfrak{p}, R / I$ itself becomes 0 , so the sequence becomes split exact by [2, Lem. 20.1], and in this case, tensoring with $(\mathscr{G} \bullet)_{\mathfrak{p}}$ necessarily preserves exactness. 


\section{ACKNOWLEDGEMENT}

We would like to thank David Eisenbud for explaining Lemma 4.5 to us.

\section{REFERENCES}

1. Corrado de Concini, David Eisenbud, and Claudio Procesi, Hodge algebras, Astérisque 91 (1982). MR680936 (85d:13009)

2. David Eisenbud, Commutative algebra with a view toward algebraic geometry, SpringerVerlag, 1995. MR1322960 (97a:13001)

3. David Eisenbud and Joe Harris, Limit linear series: Basic theory, Inventiones Mathematicae 85 (1986), 337-371. MR846932 (87k:14024)

4. Ulrich Goertz, On the flatness of models of certain Shimura varieties of PEL-type, Mathematische Annalen 321 (2001), 689-727. MR1871975 (2002k:14034)

5. Alexander Grothendieck, with Jean Dieudonné, Éléments de géométrie algébrique: IV. Étude locale des schémas et des morphismes de schémas, seconde partie, Publications mathématiques de l'I.H.É.S., no. 24, Institut des Hautes Études Scientifiques, 1965. MR 0199181(33:7330)

6. E_ Éléments de géométrie algébrique: IV. Étude locale des schémas et des morphismes de schémas, troisième partie, Publications mathématiques de l'I.H.É.S., no. 28, Institut des Hautes Études Scientifiques, 1966. MR0217086 (36:178)

7. Hideyuki Matsumura, Commutative ring theory, Cambridge University Press, 1986. MR:879273 (88h:13001)

8. Brian Osserman, Linked Grassmannians and crude limit linear series, International Mathematics Research Notices 2006, no. 25, 1-27, Article ID 25782. MR2276347 (2008d:14012)

9. _ A limit linear series moduli scheme, Annales de l'Institut Fourier 56 (2006), no. 4, 1165-1205. MR2266887 (2007h:14042)

10. Elisabetta Strickland, On the conormal bundle of the determinantal variety, Journal of Algebra 75 (1982), no. 2, 523-537. MR653906 (84g:14053)

11. Zhaohui Zhang, On moduli stacks of finite group schemes (2000), Ph.D. thesis, MIT.

Department of Mathematics, The University of Texas at Austin, 1 University Station, C1200, Austin, Texas 78712

E-mail address: dhelm@math.utexas.edu

Department of Mathematics, University of California, Davis, One Shields Avenue, Davis, CAlifornia 95616

E-mail address: osserman@math.ucdavis.edu 